\title{
Desarrollo y evidencias de validez de la Escala de Mindfulness en el Trabajo (EMT)
} para adultos costarricenses

\section{Shirley Broitman, Emerson Gaari, Diana Molina, Itzel Esquivel, Edgar Vargas, Carolina Murillo, Shirley Lewis-Bolton, Emilia García, Josiane Pawlowski}

\section{Universidad de Iberoamerica}

\section{Resumen}

Mindfulness se define como estar atento y consciente de lo que está ocurriendo en el presente. Este estudio es resultado de una práctica de estudiantes en un curso de Licenciatura. Fue desarrollada una Escala de Mindfulness en el Trabajo (EMT) y se analizaron algunas de sus propiedades psicométricas. Ochenta adultos de 20 a 50 años de edad, 55\% mujeres, contestaron la EMT y la versión mexicana de la escala de atención plena MAAS. La validez de contenido se obtuvo mediante evaluación por expertos y entrevista cognitiva. La confiabilidad fue examinada por medio del alfa de Cronbach $(\sigma=.84)$. La validez de constructo se evaluó por evidencia de homogeneidad de reactivos, resultando en 22 ítems que presentaban correlación de Spearman significativa con el total de la prueba, y por evidencia convergente mediante correlación de Pearson $(.65 ; p<.001)$ entre las dos escalas estudiadas. Los resultados sugieren que la EMT presenta evidencias de confiabilidad y de validez de constructo, pero requiere importantes modificaciones. Se presentan reflexiones sobre el proceso de construcción de instrumentos psicológicos.

Palabras clave: Mindfulness, atención plena, trabajo, validez, fiabilidad

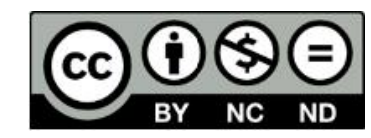




\section{Introducción}

El origen de Mindfulness proviene de la tradición Budista y su nombre original en sanscrito es smrti (léase sati), cuya traducción podría derivarse en significados como "memoria", aunque también tiene la connotación de atención y en otras ocasiones ha sido traducida como "self-possesion"; sin embargo, los autores coinciden en que sati es una de las palabras de la psicología ética del Budismo más difícil de traducir (Gethin, 2011). El nombre Mindfulness fue introducido a occidente por Jon Kabat-Zinn (2005) quien lo define como "la conciencia que surge de prestar atención, con propósito, en el momento presente y sin juzgar" (p. 24, traducido al español). Desde entonces han surgido diferentes definiciones y propuestas de traducción del término, que en español es utilizado en algunas investigaciones como atención plena (León, Martín, García, \& Felipe-Castaño, 2008; Schmidt \& Vinet, 2015).

Para esta investigación, Mindfulness se ha definido por medio de cinco elementos que se traslapan entre sí y han sido propuestos por Nilson y Kazemi (2016). Estos elementos son: 1) la atención y la conciencia (awareness), que se refiere a la habilidad de estar completamente consciente y monitoreando las cogniciones, emociones y las sensaciones corporales producidas por las influencias del entorno, y la atención es vista como un prerrequisito de la conciencia ya que esta requiere que la persona mantenga el enfoque en aquello de lo que desea ser consciente; 2) el estar en el momento presente; 3) los eventos externos que impactan el funcionamiento mente-cuerpo y que normalmente no pueden ser controlados por las personas; 4) la práctica de mindfulness para lograr la aceptación, el

\section{pesco


aprecio propios, la tranquilidad e insight; y 5) un aspecto ético que resalta la dimensión social de mindfulness por el potencial de este para favorecer la justicia, la paz y el balance ecológico en el mundo.

Desde la introducción de Mindfulness en Occidente hace treinta años, se han escrito muchos artículos que presentan sus aplicaciones y diversos efectos a nivel de la salud y a nivel mental (Liétor, Fortis, \& Moraleda, 2013). También se han desarrollado diversos instrumentos con la finalidad de medir el Mindfulness. Por ejemplo, se puede mencionar el Five Facets of Mindfulness Questionnaire (FFMQ), que en su versión española presenta 39 ítems que miden cinco factores de la atención plena: observación, descripción, actuar con conciencia, no juzgar, y no reaccionar a la experiencia interna (Cebolla et al., 2012). Otro ejemplo de instrumento es The Philadelphia Mindfulness Scale que consiste en 20 ítems que evalúan dos constructos de la atención plena: conciencia y aceptación, preguntándose por la frecuencia con la que se ha experimentado durante la última semana distintos aspectos relacionados con dichos constructos (Cardaciotto, Herbert, Forman, Moitra, \& Farrow, 2008). También León et al. (2008) crearon una escala para medir la atención plena en el ámbito escolar en España, que contiene 15 ítems de tipo Likert de cinco puntos, incluyendo afirmaciones como "Cuando voy de casa al instituto me fijo en el camino que recorro".

Además de estes instrumentos, la Mindful Attention Awareness Scale (MAAS) es una medida de auto-reporte de 15 ítems que reflejan la falta de atención a través de varios dominios, por ejemplo, cognitivo, emocional, físico, general, y miden la frecuencia en que los procesos de atención y conciencia en el momento presente ocurren en la vida diaria 
(Brown \& Ryan, 2003). Las propiedades psicométricas de esta escala fueron investigadas en España por Soler et al. (2012) y en México por López-Maya et al. (2015). El formato de respuesta es una escala de seis puntos, con un rango entre "casi siempre" hasta "casi nunca", y las puntuaciones elevadas indican mayor estado de atención plena.

Relacionado a la necesidad de la práctica del estado de Mindfulness en el cotidiano, destacase que la vida acelerada y llena de distractores puede traer para muchos profesionales una mala praxis o un desgaste cognitivo atribuido a la falta de atención a las circunstancias. El estrés laboral, la individualización del trabajo, la presión temporal y la sobrecarga de trabajo pueden generar una serie de trastornos asociados, así como asma, hipertensión, desarrollo de enfermedades infecciosas, ansiedad, fobias, depresión, entre otros (Brown \& Ryan, 2003). Para explorar el Mindfulness a nivel laboral, es importante acotar el efecto de las presiones del ambiente de trabajo cuando la persona afectada no afronta adecuadamente el estrés. Tales presiones pueden provocar desequilibrio, alteración y enfermedad y se experimentan con pensamientos, emociones y conductas de ansiedad, depresión o ira, que interfieren con el adecuado funcionamiento del individuo en todos sus contextos (Guadalupe et al., 2017).

Relacionado a ese tema, Reb, Narayanan y Ho (2013) realizaron un estudio comparativo entre el empleado consciente y el empleado con "mente ausente". El propósito de esta investigación fue investigar como el ambiente organizacional afecta de manera positiva y/o negativa la vivencia del Mindfulness en sus tareas laborales. Los autores concluyen que los empleados que practiquen Mindfulness pueden reducir el impacto de 
potenciales situaciones estresantes y observarlas sin sesgo alguno, presentan buenas relaciones interpersonales, están conscientes de lograr metas de crecimiento en su ámbito laboral y presentan mayor control en la regulación de emociones, mejorando niveles de bienestar, productividad, rendimiento y autonomía laboral (Reb, Narayanan, \& Ho, 2013).

Considerando estos aspectos, investigar el nivel de Mindfulness de las personas en el ámbito laboral puede permitir adquirir la conciencia de este y cultivarlo con el fin de disminuir la tensión, reducir el estrés, mejorar el bienestar y la salud en personas trabajadoras, junto con la posibilidad de lograr una mayor regulación emocional y más satisfacción laboral. Asimismo, se podrá ver reflejado su efecto en el rendimiento y la productividad. Además de eso, la medición del Mindfulness permite establecer el efecto de un tratamiento o de las modificaciones del entorno de trabajo. Por la importancia de la práctica del Mindfulness en el ámbito laboral, sabiéndose que es novedoso en Latinoamérica y que aún no se cuenta con una escala estandarizada para medir el constructo en Costa Rica, este trabajo tiene como objetivo crear una escala para medir el Mindfulness en personas de 20 a 50 años de edad que poseen un trabajo remunerado y evaluar algunas propiedades psicométricas relacionadas a la confiabilidad y la validez de esa escala. Es importante destacar que la presente investigación fue conducida con el propósito de: 1) colocar en práctica el conocimiento adquirido durante el curso de Seminario Avanzado en Investigación Psicológica de la Licenciatura en Psicología de UNIBE, y 2) familiarizar a los estudiantes con la creación de instrumentos de medición psicológica. 


\section{Método}

\section{Participantes}

La muestra consistió de 80 participantes en un rango de edad entre los 20 y 50 años $(M=28,33 ; D S=7,56)$. Todos presentaban ocupación laboral. Las características demográficas de la muestra se muestran en la Tabla 1.

Tabla 1. Características demográficas de la muestra

Género*

Hombre

Mujer

Nivel educacional*

Escuela

Colegio

Técnico

Universidad

Posgrado

Nacionalidad*

Costa Rica

Venezuela

Colombia

Barbados

Países Bajos $\mathrm{n}$

35

2

17

3

51

6

43.8

44

55.0

$\%$

21.3

3.8

63.8

7.5

93.8

1.3

1.3

1.3

1.3 
Ocupación*

Trabajos administrativos

y de oficina

Área de la salud

Agentes de Servicio

Abogados

8

10.0

artístico

Ámbito creativo o

5

6.3

Ámbito educativo 5

6.3

Ingenieros

5

6.3

Labores básicos (obrero, misceláneo y guarda)

$\begin{array}{ll}3 & 3.8\end{array}$

Ámbito tecnológico

2

Entrenador físico

Otros

8

10.0

Nota. * Todas las variables presentaron 1 caso "No informado" (1.3\%)

\section{Instrumentos}

Escala de Mindfulness en el Trabajo (EMT): Construida en el curso y principal variable de este estudio, el instrumento busca conocer el estado de atención plena en el ámbito laboral y se compone de 26 ítems que puntúan en una escala tipo Likert de 5 categorías, desde 1 "nunca" hasta 5 "siempre".

Escala de Atención Plena para la población Mexicana (MAAS), desarrollada por López-Maya et al. (2015): Consiste en una escala de 15 reactivos tipo Likert de 6 categorías, 
desde 1 "casi siempre" hasta 6 "casi nunca". Los estudios de la versión mexicana indicaron el índice de .89 de confiabilidad evaluada por el alfa del Cronbach y establecen la validez a partir de la validez de constructo.

\section{Procedimiento}

Este estudio es de tipo cuantitativo y correlacional (Hernández, Fernández, \& Baptista, 2010). La construcción de la escala EMT fue efectuada por estudiantes de Licenciatura en Psicología del segundo cuatrimestre de 2017, bajo la supervisión de la profesora del curso. Inicialmente se definió el constructo y sus dimensiones; posteriormente el grupo en conjunto creó los ítems a utilizar en dicha escala.

Para la construcción de la escala, se consultó a cuatro expertos en el tema, con el fin de contar con su experiencia y conocimiento en Investigación y en Mindfulness. Los profesionales han revisado la escala y han agregado sugerencias para adecuar los ítems al contenido teórico. También se utilizó el método de Entrevista Cognitiva (Smith-Castro \& Molina, 2011) que fue aplicada a ocho participantes, de manera que se pudiera recolectar más información sobre posibles correcciones. Los resultados permitieron perfeccionar la comprensión de las frases y la construcción gramatical de los reactivos. La escala resultó con 26 ítems, siendo 14 positivos y 12 negativos.

La evaluación de los participantes fue voluntaria, sin ninguna compensación por su contribución al estudio. Para la recolección de los datos, se explicaron los objetivos de la evaluación y se solicitó la firma del Consentimiento Informado; en seguida, se les pidió que

\section{pyeo.


contestaran a las escala, en primero lugar la desarrollada en esta investigación y después la versión mexicana de la MAAS. La recolección fue realizada de manera presencial y virtual. Los datos fueron insertados en una base de datos y se recodificaron los resultados de los ítems negativos para que todos fueron analizados como ítems positivos.

\section{Análisis de datos}

Todos los análisis estadísticos se llevaron a cabo con el Programa Estadístico SPSS, versión 19 (SPSS Inc., Chicago, Illinois, USA). Se realizó el análisis descriptivo de media y desviación estándar de la variable edad, y se hizo el análisis descriptivo de frecuencias para las variables género, nivel de escolaridad, nacionalidad y ocupación. Se utilizó el Alfa de Cronbach para medir el nivel de confiabilidad de la prueba. Además, se aplicó la correlación de Spearman (rho) entre cada ítem y el total de la escala para el análisis de la evidencia de homogeneidad ya que el instrumento estudiado presentaba ítems calificados en una escala de puntuación múltiple (Cohen \& Swerdlik, 2006). También se aplicó la prueba de normalidad de Kolmogrov-Smirnov para verificar la distribución de las variables de suma total de cada escala evaluada. Por último, se utilizó la correlación de Pearson para evaluar la relación entre las dos escalas. 


\section{Resultados}

Con respecto a la validez de contenido de la prueba, las sugerencias efectuadas por los expertos y los resultados de las entrevistas cognitivas permitieron realizar cambios en los ítems y mejorar su comprensión por los participantes.

La confiabilidad fue determinada por medio de la consistencia interna de reactivos, obteniéndose un coeficiente de alfa de Cronbach de .84 para la escala EMT y de 0.89 para la escala MAAS.

La validez de constructo fue investigada mediante la evidencia de homogeneidad y la evidencia convergente. Para verificar la evidencia de homogeneidad, los resultados de la correlación de Spearman ( $r h o$ ) entre cada ítem y el total de la escala se muestran en la Tabla 2, dónde se puede observar que 22 ítems presentaron correlaciones significativas con el total de la escala. Se puede verificar que los ítems con las correlaciones más altas son: Ítem 13. Mis reacciones suelen perjudicar mis relaciones con mis compañeros de trabajo, Ítem 20. Ante un evento estresante en el trabajo, pienso antes de reaccionar, Ítem 24. Me desconcentro fácilmente mientras ejecuto mis tareas laborales, e Ítem 10. Me cuesta aceptar los pensamientos y sentimientos que tengo. Aquellos que presentaron las correlaciones más bajas fueron: Ítem 2. Realizo trabajos sin pensar de forma automática, Ítem 3. Durante las tareas laborales mi mente piensa en el futuro o en el pasado, Ítem 5. Se me olvidan fácilmente las cosas por no prestar suficiente atención, e Ítem 6. Durante las horas de trabajo, dejo espacios para relajarme.

\section{psico


Tabla 2. Resultados de la correlación de Spearman entre los ítems y el total de la escala

\begin{tabular}{|c|c|c|c|}
\hline $\begin{array}{l}\text { Número } \\
\text { de ítem }\end{array}$ & Descripción del ítem & Rho & $p$ \\
\hline 1 & Uso estrategias para liberar tensión durante el trabajo & .24 & .04 \\
\hline 2 & Realizo trabajos sin pensar, de forma automática & .07 & .55 \\
\hline 3 & $\begin{array}{l}\text { Durante las tareas laborales mi mente piensa en el } \\
\text { futuro o en el pasado }\end{array}$ & .13 & .27 \\
\hline 4 & Logro que no me alteren los eventos adversos cotidianos & .30 & .01 \\
\hline 5 & $\begin{array}{l}\text { Se me olvidan fácilmente las cosas por no prestar } \\
\text { suficiente atención }\end{array}$ & .22 & .06 \\
\hline 6 & $\begin{array}{l}\text { Durante las horas de trabajo, dejo espacios para } \\
\text { relajarme }\end{array}$ & .14 & .23 \\
\hline 7 & $\begin{array}{l}\text { En el trabajo, me cuesta concentrarme en una sola } \\
\text { actividad }\end{array}$ & .58 & $<.01$ \\
\hline 8 & $\begin{array}{l}\text { Soy capaz de percibir mis emociones en el momento en } \\
\text { que ocurren }\end{array}$ & .52 & $<.01$ \\
\hline 9 & Soy consciente de mis deficiencias en el ámbito laboral & .32 & $<.01$ \\
\hline 10 & $\begin{array}{l}\text { Me cuesta aceptar los pensamientos y sentimientos que } \\
\text { tengo }\end{array}$ & .61 & $<.01$ \\
\hline 11 & $\begin{array}{l}\text { Cuando trabajo me distraigo con otras cosas ajenas a } \\
\text { este }\end{array}$ & .48 & $<.01$ \\
\hline 12 & $\begin{array}{l}\text { Puedo describir cómo me siento en el momento de } \\
\text { manera detallada }\end{array}$ & .45 & $<.01$ \\
\hline 13 & $\begin{array}{l}\text { Mi reacciones suelen perjudicar las relaciones con mis } \\
\text { compañeros de trabajo }\end{array}$ & .64 & $<.01$ \\
\hline 14 & Soy capaz de escuchar atentamente & .51 & $<.01$ \\
\hline 15 & Reconozco mi valor individual & .56 & $<.01$ \\
\hline 16 & Se me dificulta aceptar mis fallos en el trabajo & .42 & $<.01$ \\
\hline
\end{tabular}




\begin{tabular}{|c|c|c|c|}
\hline 17 & Soy consciente de mi relación con lo que me rodea & .35 & $<.01$ \\
\hline 18 & $\begin{array}{l}\text { Me doy cuenta de que soy capaz de omitir } \\
\text { distracciones }\end{array}$ & .46 & $<.01$ \\
\hline 19 & Evalúo mis acciones antes de realizarlas & .54 & $<.01$ \\
\hline 20 & $\begin{array}{l}\text { Ante un evento estresante en el trabajo, pienso antes de } \\
\text { reaccionar }\end{array}$ & .62 & $<.01$ \\
\hline 21 & $\begin{array}{l}\text { Soy capaz de monitorear mis pensamientos durante las } \\
\text { horas de trabajo }\end{array}$ & .45 & $<.01$ \\
\hline 22 & Adquiero consciencia de mis emociones de forma tardía & .60 & $<.01$ \\
\hline 23 & Me descubro a mí mismo divagando durante el trabajo & .48 & $<.01$ \\
\hline 24 & $\begin{array}{l}\text { Me desconcentro fácilmente mientras ejecuto mis tareas } \\
\text { laborales }\end{array}$ & .61 & $<.01$ \\
\hline 25 & Hago actividades laborales sin ponerles mucha atención & .59 & $<.01$ \\
\hline 26 & Logro mantenerme tranquilo en el ámbito laboral & .54 & $<.01$ \\
\hline
\end{tabular}

El test de normalidad de Kolmogorov-Smirnov aplicado para las variables de suma total de cada escala resultó no significativo para ambas, Suma EMT $(p=.20)$ y Suma MAAS $(p=.08)$, lo que indicó que la distribución de las variables se acercaban a la normal. De esa manera, para la evaluación de la validez convergente se realizó la correlación de Pearson entre la escala EMT y MAAS, obteniéndose $.65(p<.01)$.

\section{Discusión}

El presente estudio se desarrolló a partir de la práctica de un curso de la Licenciatura en Psicología en UNIBE en que se buscó crear una escala que midiera Mindfulness en las 
personas en el ámbito laboral y evaluar las propiedades psicométricas de esta escala en una muestra de costarricenses. De los resultados obtenidos, la fiabilidad de la escala (.84) se presentó adecuada, considerando como valor mínimo el .8 (Cea, 1999). Con respecto a la Escala MAAS, el resultado de .89 está de acuerdo al obtenido en la investigación con la muestra mexicana (López-Maya et al., 2015), verificándose que la escala permanece con el mismo índice de fiabilidad en la muestra costarricense.

Relacionado a la validez de constructo, de los 26 ítems creados para la escala EMT, la correlación significativa de 22 ítems con el total indica la homogeneidad de esos reactivos y que los demás deben ser revisados o excluidos. Entre los ítems que mostraron bajo nivel de correlación se encuentran el ítem 2 y el ítem 3, los cuales se relacionan con la percepción de cognición por parte del sujeto. De esa forma, es posible que las personas no tengan conciencia de lo que están pensando y les resulte difícil presentar metacognición cuando se debe analizar si la mente piensa en el futuro o en el pasado o bien si mientras se realiza un trabajo no se piensa en este.

Con respecto al ítem 5, el resultado bajo obtenido para la correlación tal vez pueda estar relacionado a la falta de especificidad del ítem, pues al decir "Se me olvidan fácilmente las cosas por no prestar suficiente atención", la referencia a "cosas" puede ser muy inespecífico. Se sugiere que ese ítem pueda ser modificado y se refiera a algo específico que se le olvide por falta de atención plena. De otra manera, el resultado de la baja correlación en el ítem 6 puede estar relacionado al acto de relajación en el trabajo. Es posible que muchos empleados consideren iluso relajarse en el ambiente laboral debido a que es un local de 
concentración y dedicación o que la práctica de relajación pudo haber sido mal-entendida, como un método de recreación, lo cual sucede en la minoría de los casos de personas que trabajan de manera remunerada. Una alternativa para esclarecer dicho ítem sería definir más específicamente lo que se espera del concepto de relajación. De eso se indica la necesidad de mejorar la escrita de estos ítems o de agregar nuevos ítems que puedan presentar correlaciones más altas con el total de la escala y mejorar su homogeneidad y fiabilidad.

Sobre la validez convergente, el resultado encontrado al comparar la escala desarrollada EMT con la MAAS fue de una correlación positiva moderada entre ellas. La escala MAAS fue elegida para la comparación con la EMT por ser una escala corta y ampliamente utilizada, validada y traducida para muchas otras lenguas, entre ellas Francés (Jermann et al., 2009), Portugués Brasileño (Barros, Kozasa, Souza, \& Ronzani, 2015), y Español, en su versión española (Soler et al., 2012) y mexicana (López-Maya et al., 2015). La versión mexicana fue la escogida por ser más parecida al español costarricense que la versión española y por presentar adecuado índice de confiabilidad en la muestra mexicana, lo que se confirmó en la muestra costarricense. La comparación con esta escala ofreció evidencias de la validez convergente de la EMT.

Entre las limitaciones de esta investigación, se debe considerar que el estudio es resultado de una práctica de un curso, o sea, ninguno de los que participaron en la creación de los ítems tenían conocimiento previo y profundizado sobre el tema Mindfulness. Además de eso, el poco tiempo disponible en el curso no permitió dedicarse al estudio sobre la teoría relacionada al constructo Mindfulness, lo que es esencial para la construcción adecuada de

\section{pyeo.


los reactivos de una prueba (American Educational Research Association, American Psychological Association, \& National Council on Measurement in Education, 2014; Urbina, 2004). Asimismo, el análisis que algunos expertos realizaron de la escala EMT no fue de modo presencial, por lo que la retroalimentación no se dio de la manera más adecuada. También fue una fuente de limitación de la investigación el procedimiento de recolección de datos, pues las escalas fueron aplicadas tanto de manera presencial cuanto por medios electrónicos, lo que impide aclarar las dudas de quienes las contestan e imposibilita la adecuada retroalimentación del investigador. Por otra parte, la muestra fue pequeña por lo que replicar esta investigación con una muestra más grande permitiría obtener datos más precisos. También el hecho de haber escalas con ítems sin contestar resultó en la pérdida de algunos casos en algunos análisis, por ejemplo, las correlaciones entre ítem y el total fueron realizadas para 72 casos.

Como sugerencias para que esta escala fuera perfeccionada, es importante mencionar que los pasos de la construcción de escalas deben seguir procedimientos rigurosos. Por lo tanto, todos los pasos realizados deberían ser mejor conducidos, con el tiempo necesario y la dedicación para generar conocimientos confiables. Así, se recomienda el estudio profundizado sobre el tema que se va a desarrollar la escala, la consulta a expertos en el área del tema de estudio y en psicometría, la realización adecuada de la recolección siguiendo un método detallado y uniforme con todos los participantes. Además de eso, otros diseños de investigación podrían ser planeados o otros tipos de análisis de datos. Por ejemplo, se podrían realizar pruebas de evidencia de cambios mediante pre prueba y post prueba después de que 
la muestra recibiese práctica de Mindfulness o se puede realizar una evidencia entre grupos distintos aplicando la escala a un grupo de personas que ya tengan práctica de Mindfulness u otro tipo de práctica que esté relacionada al aumento del estado de Mindfulness. También se podría aumentar la muestra y evaluar las calidades psicométricas de la escala mediante un análisis factorial exploratorio. Además, las muestras podrían ser diversificadas, o sea, se recomienda evaluar Mindfulness en otros grupos de trabajo, tales como los no remunerados. A su vez también se sugiere estudiar este instrumento en muestras con características demográficas más restringidas así como centrarse en profesiones específicas.

\section{Conclusión}

La presente investigación buscó desarrollar una escala para medir el estado de Mindfulness de trabajadores costarricenses. La validez de contenido de la escala se obtuvo mediante la evaluación por expertos y entrevista cognitiva, la confiabilidad por el análisis de la consistencia interna y la validez de constructo mediante evidencia de homogeneidad y evidencia de validez convergente. Este estudio permite concluir que, si perfeccionada y evaluada siguiendo etapas y procedimientos psicométricos rigurosos, la escala puede ser útil para medir la frecuencia con que las personas experimentan estados de atención plena en el trabajo, ya que los resultados preliminares apuntan evidencias de la fiabilidad y de la validez de la escala EMT para la muestra estudiada. A pesar de las limitaciones de esta investigación, lo relevante fue que en Costa Rica, hasta dónde se conoce, no hay todavía un estudio de la

\section{psico


validez de una escala para medir Mindfulness tampoco de una versión de ese instrumento dirigida al ámbito laboral.

\section{Referencias}

American Educational Research Association, American Psychological Association, \& National Council on Measurement in Education (2014). Standards for Educational and Psychological Testing. Washington, DC: AERA. American Educational Research Association, American Psychological Association, \& National Council on Measurement in Education (2014). Standards for Educational and Psychological Testing. Washington, DC: AERA.

Barros, V. V., Kozasa, E. H., Souza, I. C. W. \& Ronzani, T. M. (2015). Validity Evidence Validity of the Brazilian Version of the Mindful Attention Awareness Scale (MAAS). Psychology /Psicologia Reflexão e Crítica, 28(1), 87-95. doi: 10.1590/16787153.201528110

Brown, K. W., \& Ryan, R. M. (2003). The benefits of being present: mindfulness and its role in psychological well-being. Journal of Personality and Social Psychology, 84(4), 822848. doi: $10.1037 / 0022-3514.84 .4 .822$

Cardaciotto, L., Herbert, J. D., Forman, E. M., Moitra, E., \& Farrow, V. (2008). The Assessment of Present-Moment Awareness and Acceptance: The Philadelphia Mindfulness Scale. Assessment, 15(2), 204-223. doi: 10.1177/1073191107311467 
Cea, M. A. (1999). Metodología cuantitativa: estrategias y técnicas de investigación social. Madrid: Síntesis.

Cebolla, A., García-Palacios, A., Soler, J., Guillen, V., Baños, R. M., \& Botella, C. (2012). Psychometric properties of the Spanish validation of the Five Facets of Mindfulness Questionnaire (FFMQ). European Journal of Psychiatrics Asses, 26(2), 118-126. Recuperado de http://scielo.isciii.es/pdf/ejpen/v26n2/original5.pdf

Cohen, R. J., \& Swerdlik, M. (2006). Pruebas y evaluación psicológica: Introducción a las pruebas y a la medición. (4a.ed.). México: Mc Graw-Hill.

Gethin, R. (2011). On some definitions of mindfulness. Contemporary Buddhism, 12, 263279. doi: $10.1080 / 14639947.2011 .564843$

Guadalupe, L., Miranda, R., Soto, K., Borrero, T., Cortés, O., Scarpati, M., \& Ucrós, M. (2017). Incidencia de Mindfulness y Qi Gong sobre el Estado de Salud, Bienestar Psicológico, Satisfacción Vital y Estrés Laboral. Revista Colombiana De Psicología, 26(1), 99-113. doi:10.15446/rcp.v26n1.54371

Hernandez Sampieri, R., Fernandez Collado, C., \& Baptista Lucio, M. (2010). Metodología de la Investigación. Quinta Edición. México, D.F.: McGrawHill.

Jermann, F., Billieux, J., Larøi, F., d'Argembeau, A., Bondolfi, G., Zermatten, A., \& Van der Linden, M. (2009). Mindful Attention Awareness Scale (MAAS): Psychometric properties of the French translation and exploration of its relations with emotion regulation strategies. Psychological Assessment, 21, 506-514. doi:10.1037/a0017032

\section{pesco


Kabat-Zinn, J. (2005). Wherever you go there you are: Mindfulness meditation in everyday life. New York, NY: Hyperion.

León, B., Martín, E., García, A., \& Felipe-Castaño, E. (2008). Psicología de la Educación. Estudio Preliminar de la Escala de Atención Plena "Mindfulness" en el Ámbito Escolar. Revista Internacional de Psicología del Desarrollo y de la Educación, 1(2), 371-380.

Liétor, N., Fortis, M., \& Moraleda, S. (2013). Mindfulness en medicina. Revista Medicina Familiar Andalucía, 4(2), 166-179. $\quad$ Recuperado de http://www.samfyc.es/Revista/PDF/v14n2/v14n2_07_artRevision.pdf

López-Maya, E., Hernández-Pozo, M., Méndez-Segundo, L., Gutiérrez-García, J., AraujoDíaz, D., Nuñez-Gazcón, A., \& Hölzel, B. (2015). Psychometric Properties of the Mexican Version of the Mindful Attention Awareness Scale (MAAS). Psychologia. Avances de La Disciplina, 9(1), 13-27.

Nilsson, H., \& Kazemi A. (2016). Reconciling and Thematizing Definitions of Mindfulness: The Big Five of Mindfulness. Review of General Psychology, 20(2), 183-193. doi: 10.1037/gpr0000074

Reb, J., Narayanan, J., \& Ho, Z. W. (2015) Mindfulness at Work: Antecedents and Consequences of Employee Awareness and Absent-mindedness, Mindfulness Recuperado de http://sci-hub.io/10.1007/s12671-013-0236-4

Schmidt, C., \& Vinet, E. V. (2015). Atención Plena: Validación del Five Facet Mindfulness Questionnaire (FFMQ) en estudiantes universitarios chilenos. Terapia Psicológica, 33(2), 93-102. doi: 10.4067/S0718-48082015000200004 
Smith-Castro, V., \& Molina, M. (2011). La entrevista cognitiva: Guía para su aplicación en la evaluación y mejoramiento de instrumentos de papel y lápiz. Serie Cuadernos Metodológicos. San José, CR: Instituto de Investigaciones Psicológicas.

Soler, J., Tejedor, R., Feliu-Soler, A., Pascual, J. C., Cebolla, A., Soriano, J., ...Perez, V. (2012). Psychometric proprieties of Spanish version of Mindful Attention Awareness Scale (MAAS). Actas Españolas de Psiquiatría, 40(1), 19-26.

Urbina, S. (2004). Essentials of Psychological Testing. New Jersey: John Wiley \& Sons, Inc. 